Revista de la red interuniversitaria de estudios sobre las literaturas rioplatenses contemporáneas en Francia

7 | 2012

Arqueologías

\title{
La intimidad de una escritura
}

\section{Roxana Páez}

\section{OpenEdition}

\section{Journals}

Edición electrónica

URL: http://journals.openedition.org/lirico/556

DOI: $10.4000 /$ lirico. 556

ISSN: 2262-8339

Editor

Réseau interuniversitaire d'étude des littératures contemporaines du Río de la Plata

Referencia electrónica

Roxana Páez, «La intimidad de una escritura », Cuadernos LIRICO [En línea], 7 | 2012, Puesto en línea el 11 octubre 2012, consultado el 01 mayo 2019. URL : http://journals.openedition.org/lirico/556 ; DOI 10.4000/lirico.556

Este documento fue generado automáticamente el 1 mayo 2019.

\section{(c) $(1) \Theta \Theta$}

Cuadernos LIRICO está distribuido bajo una Licencia Creative Commons Atribución-NoComercialSinDerivar 4.0 Internacional. 


\title{
La intimidad de una escritura
}

\author{
Roxana Páez
}

\author{
Un esquema \\ el terreno (Tía "Clara") \\ el clima ("Amparo") \\ el clima y la plantita ("Choli") \\ Germinación (yo) \\ LA PLANTITA (yo)
}

Nota manuscrita de M.Puig

1 A mediados de los años noventa, junto a un grupo de colegas de la Universidad Nacional de la Plata, viajábamos cada miércoles a la capital para permanecer toda la tarde en la habitación en que la madre, el hermano y la sobrina de Manuel Puig habían almacenado sus libros y papeles. Antes de que se fuera del país natal, esa habitación había sido su cuarto propio. La biblioteca no era enorme. Los anaqueles tenían muchísimas películas copiadas por amigos y coleccionistas, libros de cine y cajas con papeles. Entre esos documentos tuve el placer de descubrir la transcripción de las grabaciones de la voz del albañil que desembocaron casi literalmente en Sangre de amor correspondido. También estaban en los estantes, los materiales inciales, entre otros, para La traición de Rita Hayworth, su primera novela.

Las líneas siguientes, consecuencia de aquellas visitas y de mis trabajos anteriores sobre la narrativa de Puig ${ }^{1}$, se publicaron en una edición interna de la Universidad Nacional de La Plata. Aprovecho la amable invitación de Julio Premat para darlas ahora a conocer. ${ }^{2}$

3 Indudablemente, el contacto con los "manuscritos" acerca al que los lee al momento de la enunciación, porque cualquier trazo de escritura lleva implícita la huella del sujeto gramatical. De manera que ante los pre-textos de cualquier novela, y sobre todo si es la primera de un escritor, deberíamos de asistir a la trasposición del sujeto biográfico en narrador de ficción.

4 Hablamos de los dactilogramas (papeles dactilografiados) y de los autógrafos (manuscritos de mano del autor) en los que Manuel Puig elaboró La traición de Rita 
Hayworth, es decir, una novela que ha generado miles de páginas críticas, entre otras cosas, por la conjunción de voces sin voz que las enuncie.

Precisamente, esos papeles se convierten en el back-stage de los conflictos enunciativos, de la producción de la escritura de Puig. No son objetos de culto, fetiches, sino objetos culturales donde se ve hacia atrás la producción textual. En palabras de Almuth Grésillon,

C'est l'écriture la plus intime, celle des cahiers et des carnets, qui montre comment, le vécu, le réel, le biographique, ont profondément partie liée avec l'écriture de l'œuvre et comment, par approximations infinitésimales et au prix de conflits cruciaux, le moi réel peut se métamorphoser en narrateur de fiction. ${ }^{3}$

6 Nosotros preferiríamos decir que esa escritura íntima muestra cómo el que escribe constituye su alteridad, objetualiza su propio yo como otro.

7 La mayor parte de esas notas figuraba en material de descarte: dorso de memos de la gerencia de Air France (donde Puig había trabajado), de hojas con membrete de sociedades de producción cinematográfica y, fundamentalmente, de las hojas escritas por él mismo pero ya "superadas" por nuevas reescrituras. Reutilizar, lo que hacían Flaubert y Zola en el siglo XIX antes de la superindustrailización del papel, en Puig "duplica" su modo de agenciar y trasvasar al lenguaje escrito (donde obviamente ya no es lo real), su modo de dar vuelta lo que ya está hecho (maniobra que se inscribe en la propia iniciación literaria).

Retomando la instancia de la enunciación, aun sin narrador, ésta se recupera sobre todo en el espacio dialógico entre el discurso ficcional y el metadiscurso, entre la situación de enunciación y el enunciado, dado por las indicaciones que se deja el escritor para proseguir la escritura la próxima vez : "La sintaxis de Toto y Mita puede ser la misma, por lo tanto... cuidar la de Delia, 'Héctor' no creo que haya que cambiar mucho, no ? y la de 'Paqui' por un tiempo -luego releer (dejar pasar un mes por lo menos)" o " $1^{\circ}$ Dejar descansar composición, $2^{\circ}$ Pasar Esther, $3^{\circ}$ Pensar Herminia (revisar papelerío al respecto)".

9 A pesar de esos micro-proyectos, en consonancia con la estética en tren de constituirse, Puig es un escritor con tendencia copista más que tachista. Hay reescrituras, supresiones, sustituciones, desplazamientos y otras huellas de los senderos que podría haber tomado el texto, pero con la prolijidad del que va "corrigiendo" en la mente, ilusión que puede dar la escritura dactilografiada, pasada en limpio permanentemente. Todo lo cual no quita la faz programática, la recapitulación y precisión de esas pequeñas listas e índices provisionales que, más bien, acompañan el proceso de expansión narrativa.

10 No obstante la ausencia de la figura del narrador, existen esas anotaciones de la mano del autor que, además de asentar automensajes en forma nominal, utiliza durante una gran parte del proceso los nombres familiares, su propio apodo ("Coco") y, en algunas circunstancias, directamente "yo" ("Germinación : yo" ; "La Plantita : yo").

11 Por acuerdo con la familia del escritor, los nombres iniciales, familiares, de algunos de los personajes fueron reemplazados en estas citas por el nombre definitivo, entrecomillado, que adquirieron como personajes de la novela.

12 Cuando en las puestas a punto, los nombres comienzan a hacerse ficcionales (sin contar que muchas veces se mantiene una alternancia entre el "real" y el "definitivo"), el disimulo ostenta: se cambian las consonantes repetidas (Toto en lugar de Coco, por ejemplo) o se mantienen las mismas iniciales y el mismo número de letras; un nombre muy común es modificado por otro igual de corriente, un apellido catalán por otro de 
igual ascendencia, o leve variación del cargo militar: "Coronel Vallejos" en lugar de General Villegas, casi anagramas imperfectos, evidentes. Si la dimensión autobiográfica adquiere su índole extratextualmente, no es tan fácil trazar la frontera cuando se trata de los pre-textos que apuntan "Capítulo papá" o "No es necesario que Adhemar vaya a 'Vallejos'. Sólo hablar y recordarlo." Por supuesto, así como no se trata de volver a los manuscritos como lo haría la filología, tampoco se intenta usarlos para la reincorporación de la biografía en los estudios literarios. Se miran con detenimiento los mecanismos de la puesta en escena de un yo, como persona, como máscara, de qué manera se opera el juego entre ficción y realidad (realidad -el yo- que se estructura, a su vez, como ficción), donde el yo resultante es producido por la palabra.

"Toto c'est moi", dice Puig en una entrevista para una revista francesa, ${ }^{4}$ así como varias veces dirá "yo soy Molina", y en cada entrevista con extranjeros : "Nací en un pueblito de la pampa...". La "equivalencia" se sustenta también en las solapas y contratapas, como para que ya desde el umbral el lector acepte esa especie de pacto fanasmático, sin que el texto efectúe retóricamente esa voluntad de identificación ${ }^{5}$, no habiendo enunciación homogénea, focalizada en Toto, ni utilización del nombre propio (ningún lector tiene por qué estar en conocimiento del apodo familiar). Como señaló Lejeune (1975), el lector entra en las novelas no sólo como en ficciones que remiten a realidades humanas, sino también a fantasmas que revelarían a un individuo. Ahora bien, no es este tipo de semejanza inverificable (en palabras del mismo autor), lo que puede interesar a una lectura crítica, sino el tipo de lectura que esa semejanza produce. Al respecto, dice Blanchot : "El relato se distingue del diario porque se enfrenta a lo que no puede ser comprobado, lo que no puede dejar constancia ni reseñarse. El relato es el lugar de la imantación que atrae la figura real a los puntos en que debe situarse para responder a la fascinación de su sombra". ${ }^{6}$ La relación de semejanza entre la figura y la sombra produce un yo mítico. Sólo en dos capítulos de La traición de Rita Hayworth irrumpe ese yo (hay un tercero donde se ransforma en el narrador de la "película que más me gustó"), y en los trece restantes las voces de la novela, sin dejar de dar cuenta de sí mismas, tienen un denominador común : Toto. A muchas voces, Toto, "hablado" y "escrito", aparece en los primeros meses de vida, durante su crecimiento y en la interrupción de su crecimiento. Incluso su genealogía iba mucho más lejos en la primera parte elidida ("Pájaros en la cabeza"), enfatizando la Argentina aluvial, babélica de las primeras décadas del sigo a través de la voz de la tía. En lugar de la confidencia personal, irrumpe el bloque de infancia, la experimentación (báscamente en los capítulos de Toto) y así todo lo que caracteriza la novela autobiográfica (el narcisismo, la indagación, la autocrítica) se vuelve absolutamente singular por la disolución del artificio que corrientemente lo sostendría.

El recuerdo dice : ¡padre, madre !, pero el bloque de infancia está en otro lado, en intensidades más altas que el niño compone con sus hermanas, sus amigos, sus trabajos y sus juegos, y todos los personajes no familiares en los cuales desterritorializa a sus padres cada vez que puede.?

14 Es en este sentido que pueden leerse las palabras de Puig en una entrevista con Saúl Sosnowski :

Yo quería que el cine fuera la realidad, y por eso las horas que no podía pasar en el cine me gustaba pasarlas contando una película, para que todo el día fuera cine. La gente que tenía tiempo para escucharme estaba bastante desocupada. La traición resulta una galería de desubicados, de gente que no encuentra su camino. De esa gente me animé a escribir porque los conocía más, habían estado más cerca mío, porque me habían dedicado tiempo. ${ }^{8}$ 


\section{Pájaros en la cabeza} "decepción técnica" con respecto al guión cinematográfico. Lo autobiográfico desempeña un papel no desestimable en el pasaje al régimen de la novela (no sólo como fracaso de un objetivo de la vida real -más allá de la vuelta a esa actividad como novelista consagrado, sino porque se encuentra la novela cuando se busca lo propio, lo "argentino") : "[...] Me acercaba a los treinta años, in extremis me aconsejaron escribir un guión que no sólo sucediera en la Argentina sino que tuviera personajes reales."9 Puig decidió hacer bosquejos con cada personaje de un episodio familiar para "poner distancia con ese material autobiográfico" y así "aclarárselos". Al castellano "lo despreciaba olímpicamente (...) era el idioma del cine subdesarrollado (...) ¿Qué hacer ? No sabía cómo describir los personajes, no encontraba el vocabulario".${ }^{10}$ Más adelante señala que no abordó la tercera persona por esa imposibilidad técnica (aunque también aclara que no era la forma en que le convenía acercarse a los personajes), sólo podía "reproducir voces". Otras justificaciones hablan de una dificultad ideológica con el propio idioma: "La tercera persona me obliga a usar la lengua castellana en su estado puro de artículo de importación"11, o, hablando de los "clásicos españoles" (probablemente se refiriera a argentinos), están "todos teñidos de pampa, de machismo". ${ }^{12}$ Pero lo más sintomático es que en la misma entrevista dice : "Me di cuenta que todo era un gran error. Que lo que yo quería era prolongar horas de espectador infantil. Reescribía alguna película que me había impresionado mucho, pero darme cuenta de esto me costó tres guiones."

En la escritura autobiográfica siempre hay una indagación que, en el proceso de estetizarse, no deja de volverse retórica : "Escribir esta novela fue tratar de comprender el porqué de este fracaso"13-dice Puig con respecto a los guiones. "No sabía nada de mi infancia, no tenía juicios formados sobre los acontecimientos, sobre los personajes. Pero sí escuchaba muy claramente sus voces, y lo principal era la acumulación de los detalles, de momentos muy reveladores"..$^{14}$ La pregunta por la propia persona va ligada al hallazgo de una fórmula (o viceversa), no hay una sin la otra inmediatamente el peso de la verdad se relativiza. Nora Catelli señala que "esta cuestión introduce la noción de proceso y perfectibilidad del dispositivo retórico de la confesión y, al tiempo, relativiza el problema de la verdad y la mentira, que se tornan asuntos literarios y no valores absolutos extrínsecos". ${ }^{15}$

Más allá de esa relatividad, hay una especie de consenso en torno a la idea, rechazada por Lejeune y sustentada por varios escritores, especialmente Gide (cuya Symphonie pastorale marcó profundamente a Puig, según sus propias palabras), de que la ficción autobiográfica dice más o es más verdadera que la autobiografía, idea a su vez refrendada por Lacan : "De otro lugar y no de la realidad es de donde la verdad extrae su garantía : de la Palabra. De ella recibe la marca que la instituye en una estructura de ficción"16. Para Paul De Man, la narración autobiográfica, al efectuarse, vela lo que desfigura, plasma la ausencia a través de una presencia únicamente tropológica.

La novela y la vida, cada una tiene su escena del desencadenamiento de la escritura. En los dactilogramas se pone en evidencia que esa voz primera de la tía platense realmente existió para luego ser diferida y diseminada en distintas voces que no la excluyen. "Pájaros en la cabeza", el título del "agradable fenómeno de la escritura automática" (cf.nota 2), es la frase que repite la madre del protagonista (luego suprimida en el texto) 
ante el gusto por el canto de la empleada doméstica, espíritu sensato que la llevó a estudiar Farmacia en lugar de Letras que era lo que realmente quería. Como umbral, el título provisorio lleva implícito el acercamiento a otro de los placeres de la madre. En bruto y sin polifonía en esas veintiséis hojas tipeadas tituladas provisoriamente "Pájaros en la cabeza", halladas en el el cuarto-escritorio, está el núcleo de la novela : tedio y cine, madre e hijo que no crece pero es buen alumno, frente al primo deportista que no es "raro", las estrellas, el chisme, la novela familiar.

En cuanto a la "escena" de escritura en la novela, la composición, sólo pone de manifiesto que no es el "logro institucional" (escolar) lo que llevará al "crecimiento". Esto si se quiere pensar la composición escolar como

... La escena arcaica, primaria, primitiva que funda el acto autobiográfico (...) Generalmente luego de encontrada se revela como el dispositivo secreto del deseo de la escritura. Su acentuación de fijeza, de cuadro, de episodio, está dada por su valor de acontecimiento. ${ }^{17}$

La redacción escolar de Toto sólo señala por inversión (en el sentido de dar vuelta y en el de ganancia a largo plazo) el acta de nacimiento de una escritura, pero para que ésta nazca, el cine se volverá subsidiario de la literatura y no a la inversa. Porque es la copia (esa culminación de los "cartoncitos pintados", lo que debe revertir. Es decir, reutilización de los recortes pero en función de un nuevo conjunto, como en un plano que llamamos real (y dentro de esta lectura fantasmática), fue el recorte de Rita Hayworth, que cabe a la "debilidad" paterna, como podría haber sido Diana Durbin que es la actriz que aparece en varios papelitos de notas para la novela y finalmente desaparece.

21 El capítulo de la composición, al poner en relación el cine y la literatura, determina una estación en la desterritorialización adolescente. Llena el vacío de la mudez de Toto que debió desplazar los juegos indebidos al orden del discurso donde se "legalizan". Pero, además, cubre el "desvío" de la iniciación amorosa, cuyos mecanismos se esbozan en las notas sueltas para el final de la novela, pero que finalmente no se resuelven, salvo por interposita persona: las escuetas revelaciones del anónimo. Éste es un texto marginal, acorde a los huecos que obtura y porque se arrima en el punto de la conclusión.

La vida de Johann Strauss por escrito, esa "escena primaria", casi de infancia, fabricada (seguramente también agregada al final y en compensación del punto crítico frustrado : el fracaso del "plan familiar") muestra un fruto posible de la educación sentimental, el mismo que lleva a la narración oral de Molina : una subjetividad fascinada por su propia emoción. En este punto el espacio autobigráfico (esa máscara que anima la ausencia) se quiebra : ¿Toto hubiera leído La traición de Rita Hayworth ? O tal vez no haya tal quiebre : ¿Manuel Puig -sino hubiera sido como autor, habría leído La traición de Rita Hayworth ?: "Me interesan todos los escritores modernos que hacen experimentos, pero raramente termino sus libros".

\section{Los nombres propios}

"A mí me interesaba que sobre todo esos personajes que yo había conocido en mi infancia me entregaran sus secretos, su intimidad."18 "Dejar Paqui", "pasar Esther", "pensar Herminia", los ayuda-memoria que Puig encolumna en papelitos muestran cómo las narraciones que proliferan son expansión de los nombres propios y expansión a su vez de la fórmula descubierta al azar: hacer hablar a la tía "Clara". Tal vez al principio, el escritor titubeó sobre el "medio" de circulación de esa voz : ¿sería oral ?, ¿sería escrita? 
La huella de esa duda es una tachadura sobre el nombre de una estrella que la la tía menciona : la enmienda dice "la Bete", sobre el nombre "Bette" (Davis). El error apuntaba un pintoresquismo de la voz, sólo perceptible por escrito, que después se suprimió, dejando la falsa impresión de la coloquialidad (en realidad, finísimo trabajo de "depuración"). Los nombres son el secreto de la escritura y encierran los secretos de una vida. Los sujetos que ambas, escritura y vida, descubren son homólogos, pero no análogos. Uno está empezando a escribir, su incumbencia y preocupación son del orden del lenguaje. El otro es presa de patrones de conducta, en el orden de la existencia de un niño, con un futuro implícito en la narración : alguna vez va a escribir.

Debo explicar algo : yo siempre escribo sobre personajes reales (creo que mi trabajo es más de investigación que de imaginación), por lo tanto al acercarme a cada personaje debo proporcionarle el modo de narrarse a sí mismo [...] Resulta muchas veces que no sé ciertas cosas de un personaje pero me apasiona saberlas, entonces basta enfrentarlo con otro para que el personaje reaccione. (La última frase se encuentra tachada en el original.)

La exclusividad que da Manuel Puig al orden referencial no se sustenta, sin embargo, en el recuerdo. Como ya se dijo, él convoca la voz como expansión de un nombre propio, mecanismo que no se oculta a los ojos del lector: deja su huella en los títulos de los capítulos, nombres todos ligados a un momento determinado (el año) y a un "clima" (la estación) o a un terreno, ya geográfico ("Vallejos", La Plata -a su vez, nombres propios), ya de escritura (diario, cuadero de pensamientos, redacción escolar). Como en Proust, el acontecimiento que ha disparado la narración ha sido la expansión de esos elementos del lenguaje, vueltos súbitamente poéticos. Se pueden encontrar en ellos las tres características que enuncia Roland Barthes en "Proust y los nombres" :

- el poder de esencialización : Vallejos o Héctor no designan más que a un referente.

- el poder de citación (que podríamos llama de condensación) : profiriendo el nombre, se convoca todo lo que éste encierra, incluso la ausencia, como es el caso de Berto.

- el poder de exploración : lo cual remite a las palabras antes citadas de Puig. Se despliega el nombre propio y aparece la voz. Se desdobla : porque renace en la escritura y es otro, como el puente tendido entre "yo" y "Toto".

Hay una escansión en ilusión/decepción del aprendizaje (armar los cartoncitos, dejar de tenerlos; jugar y tirar los vestiditos; ser uno con ella, luego perder la anuencia de la madre; escribirse, pero desde los relatos de los films, para escribir un día). Retrospectivamente, se despliegan los nombres en pos de sus "secretos" buscando la clave del propio, del que "obsesiona".
1. el terreno (Tía "Clara")
2. el clima ("Amparo")
3. el clima y la plantita ("Choli")
4. Germinación (yo)
LA PLANTITA (yo) 


\begin{tabular}{|c|}
\hline $\begin{array}{l}1^{\circ} \text { Dejar descansar } \\
\text { composición } \\
2^{\circ} \text { Pasar Esther } \\
3^{\circ} \text { Pensar Herminia } \\
\text { (Revisar papelerío } \\
\text { al respecto ) }\end{array}$ \\
\hline
\end{tabular}
recibidas en que se erige su voz. Y, además, los principales constituyen una "onomástica" organizada en base a la proximidad o alejamiento económico del protagonista. En la primera novela de Puig, y pese a la diferencia entre sí, una cosa son 
Toto, Teté, Mita, y, otra, Herminia, Esther, Héctor. E incluso existe el extremo, grotesco casi, del apodo, de índole absolutamente referencial, entre personajes de segundo plano: la Pocha, la Ñata, la Rulo. El apodo frente el nombre a secas. Pero además los diminutivos entrañan otras posibles aproximaciones : Alicita, Paquita, Cobito.

\section{Padres}

En una entrevista, Puig señala la importancia de la figura paterna en la estructura de la primera novela :

Yo creo que toda la clave de la novela está en la ausencia del padre. El padre nunca está, nunca interviene. Este chico, que soy yo, siente desde el primer momento que el padre no está. Entonces se me ocurrió que si bien el padre debía figurar en la novela tenía que ponerlo al final. De ese modo el lector debía repetir la experiencia del protagonista. Le escamoteaba ese personaje que recién aparece al final. El lector re-vive la experiencia del protagonista que es la búsqueda de una figura que no está en ninguna parte y que recién se da al final. ${ }^{22}$

Como vemos abajo en la representación de la hoja de bloc, la carilla número 22, la decisión que menciona Puig, fue tomada ya avanzada la escritura de la novela. En el proyecto bosquejado, el protagonista, aunque "chiquito", ya va al cine y "le hace compañía a la madre". La carta no estaba pensada, entonces, con fecha de 1933. La modificación requiere una variación en serie, ya que el capítulo de Berto es consecutivo de lo que sucede en el segundo. Por otra parte, Puig dijo que los dos primeros capítulos los "tiró a la basura" y que el tercero pasó a ser el sexto.

La ausencia del padre, sin embargo, es la de su voz, de su "presencia", ya que Berto es nombrado desde el primer capítulo édito, así como en las hojas halladas con esa "voz de tía", tituladas "Pájaros en la cabeza". En los apuntes, lo paterno obsesiona, pero no se textualiza. Una nota adjunta a una escritura primera del capítulo $\mathrm{V}$, pone de manifiesto esta preocupación con una gradación de padres :

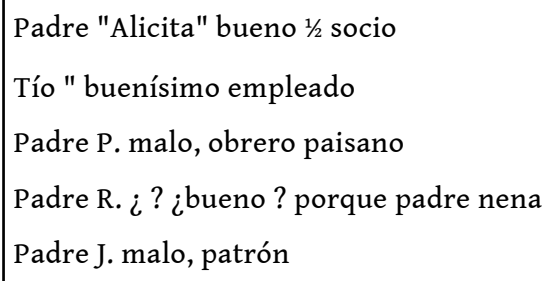

En primer lugar, llama la atención la "valoración" unida a la posición en el mercado laboral, lo que además no la determina. En los apuntes tomados en hojas de bloc, hay un "padre (de) Adhemar" vinculado con Dios y, al parecer, la culminación de la narración. 




"V.", según se aclara en otra hoja del bloc, es la inicial del apellido de una de las niñas mencionadas en el capítulo del "anónimo". La "chanchada a la Paqui" se refiere a la delación que hace Toto de su amiga Paqui, al novio de ésta, sobre las "experiencias previas" de su novia.

El padre de Toto, Berto, ausente en esa catalogación, es analizado permanentemente de un modo "omnicomprensivo", lleno de justificaciones : "papá y bondad con empleados" ; "necesidad de hablar mal del padre, inventando... (en un $35 \%$ ), "arquetipo del casado jovencito/ un padre niño" ; "papá un volcán que puede explotar en cualquier momento (... ) papá ocupadísimo, preocupadísimo... caricias". También está el padre de Herminia ("Papá y Schubert tenían razón al vivir por la belleza que es la última esperanza") y en la versión édita, el de Paqui, que azota por una nimiedad y se vuelve comprensivo ante la "falta".

Los padres confluyen en el padre idealizado, ${ }^{23}$ inspiran bondad y temor : "y en la mañana oscura parado al lado mío con la cara negra, de papá de carbón, del gitano de noche de miedo de caerme de la cama", dicen otras notas. El "motivo" del gitano, reescrito varias veces en éstas, se disemina en el tercer capítulo, rompiendo el destello de la identificación con el padre, aunque asociado a él. En las notas, la imagen del padre (o del vigilante) en la vidriera apagada de la juguetería se reescribe hasta asociarse con los viajes en tren de "Vallejos" a La Plata, en los que madre e hijo duermen solos en el camarote. En la reescritura definitiva, la fantasía del gitano empieza antes del fin de la fiesta a la que han concurrido. El padre se niega a acompañarlo al baño, que lo haga una nena, $i$ acaso no va siempre con la madre al baño de mujeres? Toto piensa en la posible oscuridad del baño 
en el que podría aparecer el gitano que roba los chicos (otra expansión narrativa, en este caso derivada de una suerte de mito racista popular) y más adelante, por el mismo rechazo del padre, en la posibilidad de escaparse cruzando la plaza hacia donde está su madre, donde también podría irrumpir el gitano. Luego aparece, como en las notas, asociado al vigilante y al reflejo del padre, pero sin la alusión al camarote del tren. Por último, el temor se proyecta en la nena en el mismo baño que, siguiendo el hilo de la fantasía infantil, el gitano "viola" y "come". La idea del "rapto" asociada, al mismo tiempo desterritorializa a Toto de la novela familiar. El temor y la voluptuosidad de la fuga son las dos caras del rapto, como en Pinochio de Collodi : "Mamá me busca y no me encuentra y va a buscarme al baño del cine, pero no estoy en el baño de las mujeres y al baño de los varones no puede entrar !". Respecto del rapto, los personajes Paqui y Mita han leído $E l$ hombre que ríe de Victor Hugo, que lo tiene como tema.

La madre no puede encontrarlo. No hay pertenencia a los territorios conocidos, aunque de alguna manera el protagonista busca "recuperarlos".

En el origen de la fantasía, está la pura referencialidad: los gitanos aposentados en la zona del parque de "Vallejos" y la seducción del gallego gitano, López Lagar, actor de radioteatro, mencionado en el dactilograma "Pájaros en la cabeza". La arbitrariedad de la lectura, no obstante, permite ve el crecimiento múltiple en la "ficcionalización" de ese origen.

41 La edipización (dormir con la madre), la amenaza de castración (la cara negra que de pronto irrumpe en el vidrio) "animan" un bloque de infancia desde la "adultez" del narrador ausente (oculto) que no ignora el relato psicoanalítico : ahí está varias veces el fantasma de las notas. Como dicen éstas, un día Toto ya no puede contarle todo a la madre, y entonces escribe independientemente del vínculo epistolar con ella. Pero escribir, antes que la asunción de la ley paterna que flaqueaba, es seguir una línea de fuga donde las prohibiciones se suspenden.

"Padre Adhemar" no llegó a la conclusión de la novela. El padre que aparece finalmente en el capítulo de Herminia es el de la profesora de piano, a partir de un comentario sobre la vida sórdida de Schubert. Las notas aludidas, que pertenecen a un estado avanzado de su desarrollo, dan cuenta de una "estratificación moral" condensada en la palabra de Herminia. Por un lado, el mal achacado al sexo, ligado con cierta violencia "animal", ejercida sobre alguien menor, débil (motivo que se repite en Boquitas pintadas con Juan Carlos), como una chica de doce años, o tres contra uno, o con la fealdad, por ejemplo la de Yamil. En el otro extremo, aparece la ética que devuelve una estética: Schubert, Herminia, Toto, Adhemar. "... Y la belleza ? Papá y Schubert tenían razón al vivir por la belleza, que es la última esperanza" (nota de bloc). Cabe preguntarse si esto fue pensado en un momento como final y no simplemente del capítulo "Cuadernos de pensamientos de Herminia, 1948). Terminarla con el "capítulo papá" fue una decisión posterior.

Habría habido una primera etapa de escritura a la que pertenece "Pájaros en la cabeza". En una etapa más avanzada, ya diseminado y desarrollado ese primer esbozo, el "capítulo papá" aparece como consecutivo del que quedó como segundo.

La conjunción ético-estética de la primera novela de Puig recuerda el final de El beso de la mujer araña, en cuya contraposición de valores, dicha noción estaría representada por Molina, finalmente "la heroína", frente a la "claridad" bien pensante de Valentín. 




En la nota original aparece el nombre real de la tía, hermana del padre de Puig, luego transformado en Adela. También se observa ya la idea general de la versión final de la carta y su función en la novela, pero con algunas leves variantes: la carta de Berto es llevada más atrás en el tiempo, al año 1933 cuando Toto es un bebé de ocho meses. Manuel Puig había nacido el 28 de diciembre de 1932. El momento elegido para la carta de Berto a su hermano marca más el contraste entre una situaición idílica -padre, madre, niño, glamour de Sagrada Familia en una estampa- y la situación económica precaria y sacrificada, cuya responsabilidad recae casi absolutamente en la figura paterna. Pero su eficacia reside, fundamentalmente, en que redondea la estructura, creando una equivalencia entre principio y final. Además es un gesto "moderno" para el discurso, además de poético y equivalente a las cartas que se queman al final de Boquitas pintadas. Es decir, la carta de Berto puede ser leída por el lector, a pesar de que el personaje decide que ésta no llegue a destino. "Se une lo cercano con lo lejano, el pasado y el presente, el sueño y la realidad", aunque la diferencia entre ese pasado y ese presente "vacíe los recuerdos y los haga inutilizables". ${ }^{24}$ Lo más llamativo es que el bosquejo directamente aparezca como "Capítulo papá". La carta de Berto al hermano aparece como "una carta al padre", pero al mismo tiempo exonera la figura paterna sustentada por él mismo, poniendo de manifiesto la impotencia para servir de agente con respecto a la ley que debiera hacer cumplir.

La "mala palabra" no aparece en la versión definitiva. Su poda señala una vez más la depuración retórica y sutil que lleva a cabo Puig. Se borra el exabrupto que se había colado desde la subjetividad de la enunciación. No hay la misma necesidad de "limpieza" con respecto a la historia, a la novela familiar.

\section{BIBLIOGRAFÍA}

Barthes, Roland. "Proust et les noms". Le Degré zéro de l'écriture. Nouveaux essais critiques. París : Seuil, 1972.

Blanchot, Maurice. "El diario íntimo y el relato". El libro que vendrá. Caracas : Monte Avila, 1992.

Carrera, Arturo. Nacen los otros. Buenos Aires : Beatriz Viterbo, 1993.

Catelli, Nora. El espacio autobiográfico. Barcelona : Lumen, 1991. 
De Man, Paul. "Autobiography as De-facement". The Rhetoric of Romanticism. Nueva York:

Columbia University Press, 1984.

Deleuze, Gilles et Guattari Felix. Por una literatura menor. México : Ediciones Era, 1978.

García, Germán. Nanina. Buenos Aires : Jorge Alvarez, 1968.

Grésillon, Almuth. Éléments de critique génétique. Lire les manuscrits modernes. París : PUF, 1994.

Lejeune Philippe. Le Pacte autobiographique. París : Seuil, 1975.

Páez, Roxana. Del pop a la extrañeza. Buenos Aires : Almagesto, 1995.

Panesi, Jorge. "Las relaciones peligrosas", Revista iberoamericana 125, octubre, diciembre 1983.

Puig, Manuel. El beso de la mujer araña (1976). Barcelona : Seix Barral, 1982.

Puig, Manuel. La traición de Rita Hayworth (1968). Buenos Aires : Seix Barral, 1993.

Quiblier, J.-Michel y Joecker J.-Pierre. "Entretien avec Manuel Puig". Masques. Revue des

homosexualités 11, París, 1981.

Rosa, Nicolas. El arte del olvido. Buenos Aires : Puntosur, 1990.

Sosnowski, Saúl. "Entrevista". Hispamérica 3, año 1, 1973, p. 69-80.

\section{NOTAS}

1. R. Páez. Manuel Puig. Del pop a la extrañeza. Buenos Aires : Almagesto, 1995.

2. M. Puig. Materiales iniciales para la traición de Rita Hayworth, publicación núm. 1 del Centro de Estudios de Teoría Literaria de la Universidad Nacional de La Plata, 1996.

3. A. Grésillon, Éléments de critique génétique. Lire les manuscrits modernes, París : PUF, 1994.

4. J. M. Quiblier, J. P. Joecker," Entretien avec Manuel Puig". Masques. Revue des homosexualités 1, París, 1981, p. 20-32.

5. Como sí es el caso de Nanina de Germán García, novela del mismo año, publicada en la misma editorial que La traición de Rita Hayworth.

6. M. Blanchot, Maurice." El diario íntimo y el relato". El libro que vendrá. Caracas : Monte Avila, 1992.

7. G. Deleuze, Gilles y F. Guattari, Por una literatura menor, México : Ediciones Era, 1978, p. 113-114.

8. S. Sosnowski," Entrevista", Hispamérica 3, año 1, 1973, p. 69-80.

9. Ibid.

10. Ibid.

11. Respuestas dactilografiadas a una entrevista, encontrada entre sus papeles sin más datos, aunque datable por la mención de la preparación de su tercera novela.

12. Sosnowski, op. cit.

13. Ibid.

14. Quiblier, op. cit.

15. N. Catelli, El espacio autobiográfico, Barcelona : Lumen, 1991, p. 85-86.

16. Citado por J. Panesi," Las relaciones peligrosas", Revista iberoamericana 125, octubre-diciembre 1983. .

17. N. Rosa, El arte del olvido. Buenos Aires : Puntosur, 1990, p. 59.

18. Sosnowski, op. cit.

19. Panesi, op. cit. 
20. A. Carrera, Nacen los otros. Buenos Aires : Beatriz Viterbo, 1993.

21. R. Barthes," Proust et les noms". Le Degré zéro de l'écriture. Nouveaux essais critiques. París : Seuil, 1972, p. 177.

22. Sosnowski, op. cit., p. 72.

23. Panesi, op. cit.

24. R. Páez, Roxana. Del pop a la extrañeza. Buenos Aires : Almagesto, 1995, p. 44.

\section{RESÚMENES}

Las notas de un bloc, las hojas sueltas que acompañan el proceso de elaboración de la primera novela de Manuel Puig son la escritura íntima donde se ve la huella de la transformación de ciertos materiales autobiográficos en ficción. En La traición de Rita Hayworth, novela de iniciación, la cuestión resulta todavía más interesante, porque es con la que Manuel Puig se inicia como autor, pero también porque es una novela sin narrador. Las notas halladas muestran el back-stage de los conflictos enunciativos durante la gestación del libro, a veces más próximas a una investigación, como arqueología de la voz, que a la construcción ficcional. Más que en sus libros siguientes, el autor es un agente de la mutación epistemológica de su propia historia.

Les notes d'un cahier ou les feuillets volants qui accompagnent le processus d'élaboration du premier roman de Manuel Puig, gardent la trace de l'écriture intime, où l'on voit le changement des matériaux autobiographiques en fiction. Dans La trahison de Rita Hayworth, roman d'initiation, la question est encore plus intéressante : c'est le roman qui marque le début littéraire de son auteur, mais c'est aussi un roman sans narrateur. Les notes des archives montrent les coulisses des conflits d'énonciation qui surgissent lors de la gestation du roman. Ce sont des notes parfois plus proches d'une enquête que d'une construction fictionnelle, qui fonctionnent souvent comme archéologies de la voix. Plus encore que dans les romans qui suivent, l'auteur devient ainsi agent de la mutation épistémologique de sa propre histoire.

Handwritten notes in a block, as well as some lose pages that come along with the process of the development of Manuel Puig's first novel appear as intimate writing. We can read in them traces of the transformation of autobiographical material into fiction. In Betrayed by Rita Hayworth, a bildungsroman, the question becomes more interesting, because it is Puig's first publication; in order words, he becomes author with it. But this novel hasn't got a narrator. Manuscript notes show the backstage of conflicts of enunciation during the work in process : it is an archaeology of voices that becomes sometimes closer to an enquiry than to a construction based on invention. Much more than in his following books, in this novel the author becomes an agent of the epistemological mutation of his own history. 


\section{ÍNDICE}

Mots-clés: Manuel Puig, littérature autobiographique, tapuscrits, roman d'initiation, fictionalisation, avant-textes, cinéma et littérature, scène originale, nom propre, journal intime, roman familial, minorités sexuelles et littérature, roman latino-américain du XXe siècle, roman argentin des années 1960

Keywords: Manuel Puig, autobiographical literature, unpublished typescripts, bildungsroman, fiction vs non-fiction, cinema and literature, sexual politics in literature, XXth century LatinAmerican novel, Argentine novel of the sixities

Palabras claves: Manuel Puig, literatura autobiográfica, dactilogramas, novela de iniciación, ficcionalización, pre-textos, cine y literatura, escena original, nombre propio, diario íntimo, novela familiar, minorías sexuales y literatura, novela latinoamericana del siglo XX, novela argentina de los ‘ 60

\section{AUTOR}

\section{ROXANA PÁEZ}

Ecrivain. Université Stendhal - Grenoble 3 